\title{
THE DIAGNOSIS OF CARDIAC SHUNTS BY INTRAVENOUS ANGIOCARDIOGRAPHY
}

\author{
BY \\ JOHN LIND, ROWENA SPENCER, AND CARL WEGELIUS \\ From the Pediatric Clinic of the Karolinska Institute (Prof. A. Wallgren) and the Wenner-Gren \\ Cardiovascular Research Laboratory, Norrtulls Hospital, Stockholm \\ Received July 6, 1954
}

In the study of cardiodynamics, the role of specific radiographic techniques is assuming increasing significance. The development of kymography and electrokymography results from the effort to record the deviations in individual portions of the cardiac silhouette as correlated with the electrocardiogram. However, the movements of the silhouette fail to give an accurate representation of the work of the heart, for, to a large extent, the activity actually takes place within the heart and cannot be visualized from outside.

Angiocardiography is, therefore, a step forward towards clarification of the total mechanical functioning of the heart. It represents a distinct addition to the armamentarium of cardiophysiology, because by this means successive contrast filling of the individual chambers and great vessels determines variations in function as well as outline. Henceforth these alterations in function, instead of being a final diagnosis, may be used to facilitate the diagnosis of specific congenital and acquired heart disease.

The internal working of the heart and the individual chambers can best be illustrated by the study of shunts; here all action takes place within the walls of the vascular apparatus and cannot be analyzed by the most perfect representation of the movements of the cardiac silhouette. Angiocardiography is another milestone along the road to better understanding of the function of this vital complex mechanism.

The normal findings in the right and left anterior oblique views are shown in Fig. 1 for comparison with the subsequent figures demonstrating shunts.

Technique. The angiocardiograms reported here were made on infants and small children under comparable and reproducible conditions. A 2.5 per cent solution of avertin, $0.1 \mathrm{ml}$. per kilogram of body weight, was used for basal anæsthesia. The radiograms were taken simultaneously in both the right and the left anterior oblique projections at a speed of 10 to 12 exposures a second in each projection. An automatic marker recorded the exact moment of exposure of each film on the electrocardiogram which was taken during the angiocardiogram. The contrast medium was 70 per cent umbradil (Astra); 1 to $1.5 \mathrm{ml}$. per $\mathrm{kg}$. of body weight was injected rapidly through the malleolar vein in infants and through the antecubital vein of the small children.

\section{Right-TO-LEFT SHUNTS}

The demonstration of any shunt is dependent on a difference in radio-opacity between two chambers of the heart, and this difference in density is dependent upon the inflow of contrast material. If the contrast material arrives at the caval orifice during atrial systole, when this opening is functionally closed, it is temporarily stored in the vena cava. Then when diastole begins, a sudden influx of concentrated contrast completely opacifies the atrium enough to visualize an existing shunt. Likewise, if it reaches the heart at the beginning of diastole when the atrium is relatively empty, there will be minimal dilution of the contrast and good opacification of the chamber. This holds 

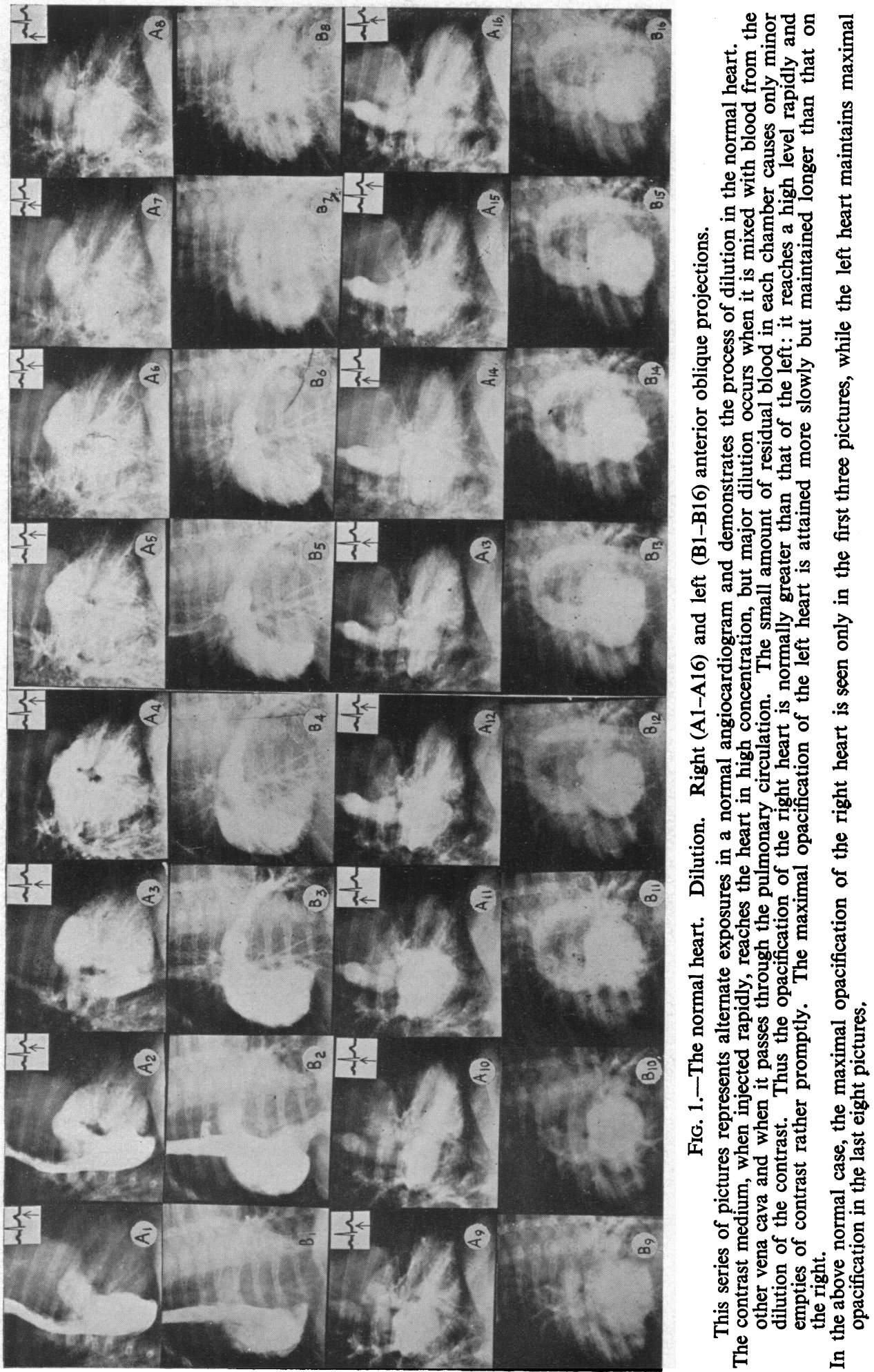
true for an atrium of relatively normal size; in a greatly dilated atrium, incomplete emptying may leave non-opacified residual blood which will dilute the contrast material and result in unsatisfactory opacification. On the other hand, should the contrast material arrive in middle or late diastole, the atrium will already be partially filled with blood, and its subsequent dilution will show insufficient opacification of the atrium to visualize a right-to-left shunt. The same holds true for the visualization of the right ventricle; the earlier in ventricular diastole the contrast reaches the right ventricle, the more complete will be the opacification. It should be emphasized that failure to visualize a shunt during the first cardiac cycle after injection may be the result of the contrast medium reaching the heart at an inopportune time.

\section{INTERATRIAL SHUNTS (Fig. 2 and 3)}

A right-to-left interatrial shunt is the ideal one to demonstrate angiocardiographically, because there is a maximum difference in the concentration of contrast medium on the two sides of the heart at the beginning of the study. When the contrast medium reaches the right atrium, no other chamber of the heart is opacified, and it streams through the interatrial defect into a left atrium which is radiographically empty (Fig. 2).

An axial view of the interatrial septum is necessary to visualize a small shunt. If the plane of the septum is directly " end on," a small jet of contrast medium from the right to the left atrium will be plainly visible, but if the interatrial septum is viewed obliquely, the opacification of the right

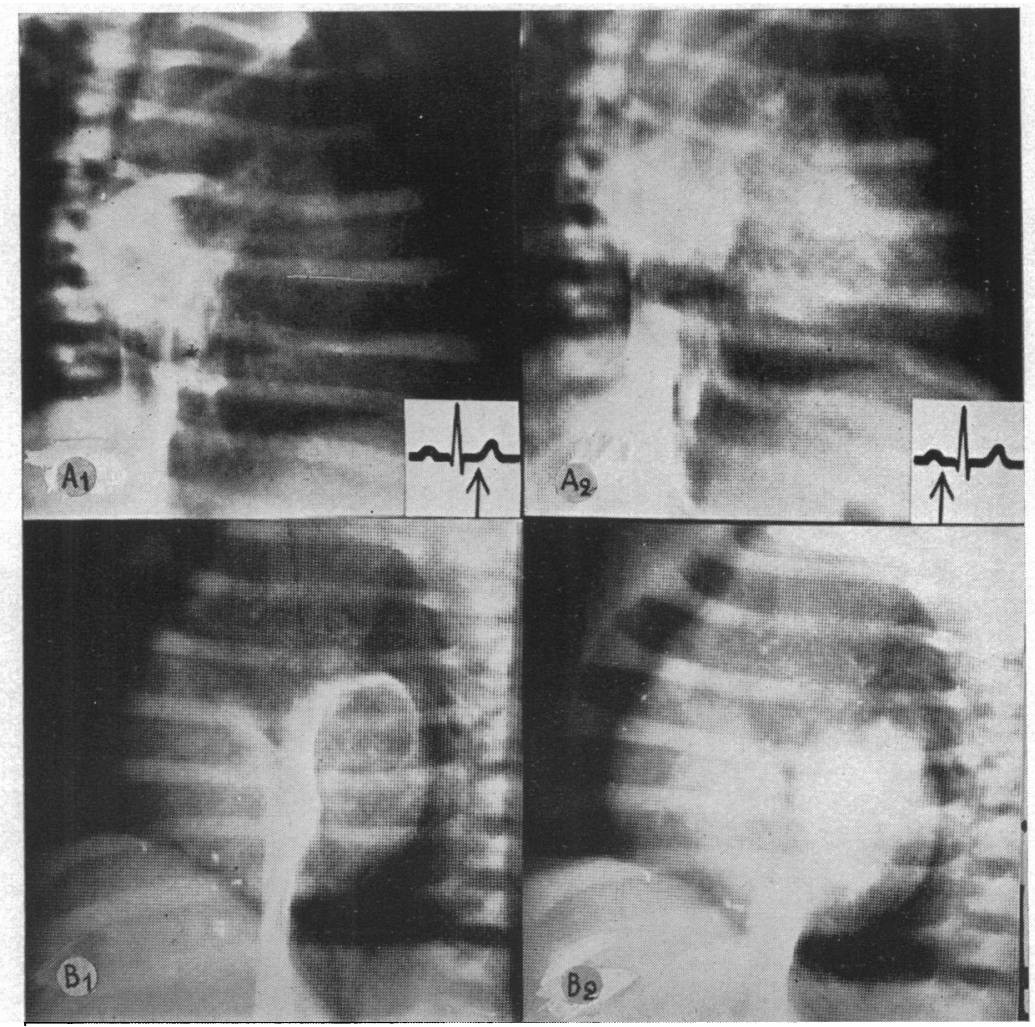

FIG. 2.-Atrial septal defect associated with pulmonic stenosis. Right-to-left shunt. Premature visualization of the left atrium. Right (A1-A2) and left (B1-B2) anterior oblique projections.

Both atria are filled in atrial diastole and have approximately the same concentration of contrast, indicating a large shunt. 

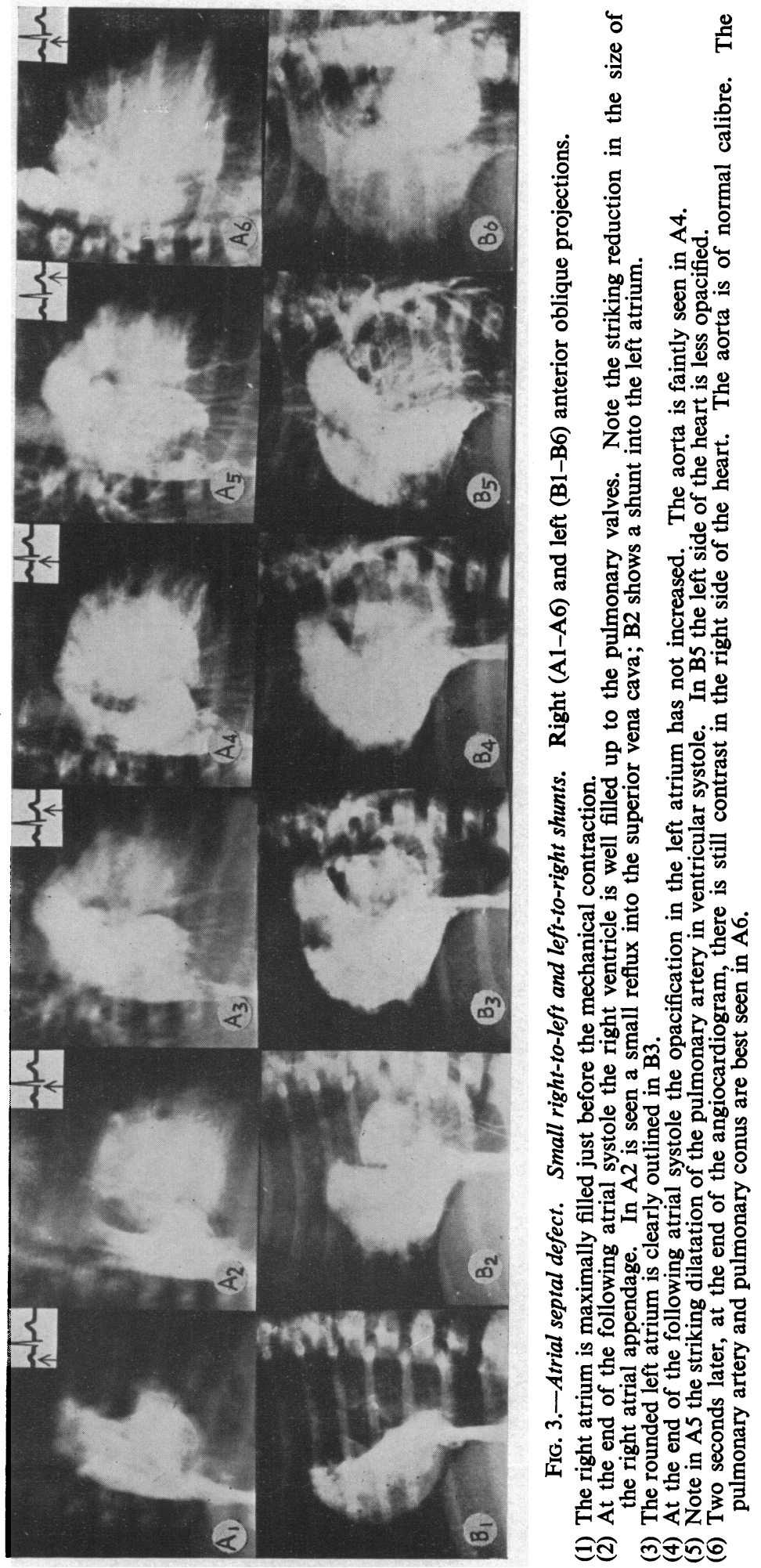
atrium may obscure a small amount of contrast streaming into the left side of the heart. The contrast medium is most concentrated as it emerges from the septal defect into the left atrium, and it is this area close to the septum which would be superimposed by the shadow of the right atrium if a true "lateral " view of the interatrial septum were not obtained.

Atrial Systolic Shunts. During atrial systole the orifices of both caval veins are functionally closed, so forceful injection of contrast medium will not materially alter the atrial systolic pressure, except inasmuch as it may have caused an increased filling in the previous diastole. This latter factor becomes less important as the heart rate decreases, because of the following facts: during early atrial diastole, when the A-V valve is closed, the forceful injection of contrast material may cause an increased filling of the atrium, but as soon as the A-V valve opens, the atrial content is spilled rapidly into the ventricle. Further atrial filling continues for a short time after the valve opens and until the atrial systole begins and functionally closes the caval orifices again. This last period, atrial diastole with an open A-V valve, is relatively short but becomes longer as the heart rate decreases, thus allowing more of the increased atrial volume to be emptied into the ventricle. Following this line of reasoning, it is obvious that the rapid injection of contrast medium may not materially alter the atrial systolic pressure, except possibly in a very rapid heart rate, and therefore is not likely to cause false visualization of a right-to-left atrial systolic shunt (as for example through a patent foramen ovale).

Atrial Diastolic Shunts. During atrial diastole the orifices of the venæ cavæ are open, so if contrast material is injected more rapidly than it can be handled by the atrium, the excess fluid injected may flow into the vena cava other than the one through which the injection takes place. The other vena cava thus acts as a safety valve. This mechanism functions only so long as the A-V valve is closed; when this valve opens the increased volume arriving at the atrium is immediately spilled into the ventricle. Thus, it becomes apparent that the rapid and forceful injection of contrast medium may increase atrial diastolic pressure during ventricular systole.

The passage of contrast material through a patent foramen ovale or a true septal defect, therefore, does not necessarily indicate the presence of a true right-to-left atrial diastolic shunt. The direction of the shunt is dependent upon the relative pressures in the atria. Normally the pressure is slightly higher in the left atrium throughout the entire cardiac cycle. If there is only a small difference in pressure in the two atria, forceful injection of the fluid may cause a reversal of the shunt during atrial diastole. This reversal in the direction of flow may occur " normally" at some point in the cardiac cycle; mixed shunts are frequently demonstrated by cardiac catheterization.

Concerning a patent foramen ovale, the chances of artificially visualizing this opening decrease markedly as the infant grows older, particularly after the first few weeks, because of the pressure in the left atrium increasing during this time.

On the other hand, a true right-to-left atrial diastolic shunt may fail to visualize if the contrast medium reaches the right atrium during late diastole, that is, after the A-V valve has opened. In this case, the contrast medium may pass through the atrium directly into the ventricle.

Right-to-left interatrial shunts are generally easy to recognize. One important anomaly with which they can be confused is abnormal venous return, as, for example, when a left superior vena cava empties into the right atrium. This vessel may be visualized in the same manner that contrast material flows into the normal vena cava during a forceful injection of the dye. Interatrial shunts will not be confused with interventricular shunts if the angiocardiogram is made in two planes synchronously; the right anterior oblique projection will show lack of opacification of the ventricles and thus exclude a shunt between the chambers.

\section{INTERVENTRICULAR SHUNTS (Fig. 4)}

Because of several factors, visualization of an interventricular septal defect may not always be completely satisfactory. On the way to the ventricle the contrast medium has already passed through one chamber of the heart and has therefore become a little more dilute. To obtain a good opacification of the right ventricle there must be a high concentration in the right atrium from the 
beginning of the ventricular diastole, because the early filling of the ventricle is very rapid. At the end of the ventricular systole the residual blood will dilute the contrast medium as it arrives. Therefore maximal opacification of the right ventricle is not obtained for two or three cardiac cycles.

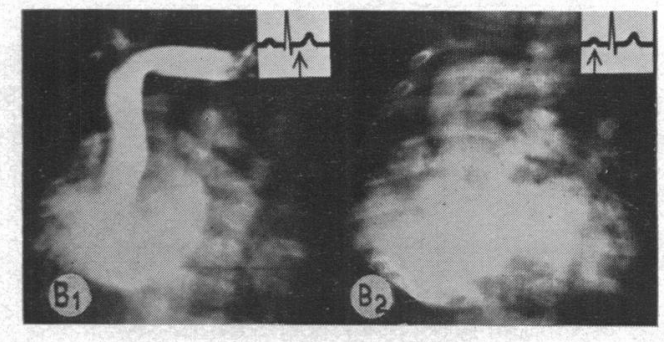

Fig. 4.-Ventricular septal defect. Right-to-left shunt. Premature visualization of the left ventricle. Left (B1-B2) anterior oblique projection.

B1. The right atrium is well filled in atrial diastole.

B2. In the following atrial systole the contrast spreads out in both ventricles.

Here, too, a shunt is not visualized as a sharp jet of concentrated contrast medium into a chamber empty of this, but usually as a simple diffuse opacification of the left ventricle. This is due to the dilution of the contrast and the more turbulent current that results in a more rapid and complete mixing.

The visualization of an interventricular shunt may also depend on the localization of the defect; in the presence of a high interventricular defect or an overriding aorta, the shunted blood (and contrast) may pass directly into the aorta without actually entering the left ventricle. Here the diagnosis will be made from the early visualization of the aorta, instead of by opacification of the left ventricle.

Since the pulmonary circulation time approximates the time required for three to six ventricular systoles, the diagnosis of a right-to-left shunt is best made during the first three cardiac cycles after the injection of the contrast medium (with a heart rate of 180,3 cardiac cycles $=1$ second); otherwise the contrast medium which has passed through the pulmonary circuit and entered the left heart will obscure the jet of contrast from right to left through the septal defect.

It is worthy of note that early visualization of the aorta may be diagnostic of a small right-to-left shunt, either atrial or ventricular. This is best observed in the right anterior oblique projection where the shadow of the ascending aorta is superimposed on that of the descending aorta. In the left anterior oblique view, where the two limbs are separated, the small amount of dye in the aorta may be invisible, but when the two limbs are superimposed, it may be possible to outline the vessel.

It should be emphasized that the size of a shunt may be roughly estimated by comparing the degree of opacification in the two sides of the heart or in the pulmonary artery and the aorta.

\section{Patent Ductus Arteriosus}

A right-to-left shunt through a patent ductus arteriosus has been described in adults, children, and newborn infants. It is easy to diagnose angiocardiographically because early filling of the descending aorta is pathognomonic. The distal aorta visualizes simultaneously with the pulmonary artery but the ascending and transverse aorta remain invisible. This diagnosis can be confirmed by the lack of contrast filling of the left side of the heart on the left anterior oblique projection, i.e. absence of other right-to-left shunt. When there is no other right-to-left atrial or ventricular shunt, early opacification of the distal aorta can result only from flow of contrast material from right to left through a patent ductus arteriosus. 


\section{LEFT-TO-RIGHT SHUNTS}

A new principle of diagnostic radiology is the study of the dilution of contrast material. Heretofore, the primary aim of angiocardiography has been to obtain the strongest possible concentration of contrast in the heart, but a critical evaluation of dilution, the opposite of concentration, will enrich the field of cardiodynamics.

A prerequisite in the study of dilution is a rapid exposure technique that permits the comparison of successive cardiac cycles. It is necessary to compare the opacification of various chambers in the same cycle and the same chamber in various cycles, and only a rapid exposure rate will provide sufficient exposures to make this comparison.

A previous knowledge of the normal dilution can be gained from angiocardiograms made under comparable conditions of anæsthesia and speed of injection of contrast (Fig. 1). With this background, it is possible to evaluate abnormal dilution of contrast in the presence of a shunt.

A left-to-right shunt is characterized by a prolonged opacification of all the chambers of the heart, a continued visualization of the blood that is withdrawn from the systemic circulation and recirculated through the heart and pulmonary vessels. This short-circuited blood increases the volume that must be handled by the affected chambers and results in dilatation of these chambers. An anatomic consequence of this decrease in the volume of the systemic circulation is the comparatively small calibre of the aorta, and as a result of the concomitant increase in the volume of the lesser circulation, there is a profuse pulmonary vascularity.

\section{INTERATRIAL SHUNTS (Fig. 5)}

When the contrast material reaches the heart, a significant left-to-right shunt will cause an obvious dilution in the right heart; there is a discrepancy between the heavy opacification of the vena cava and the poor filling of the right atrium. If this dilution can be seen to occur during atrial systole it is positive evidence of a shunt, but it may result during diastole from mixing of
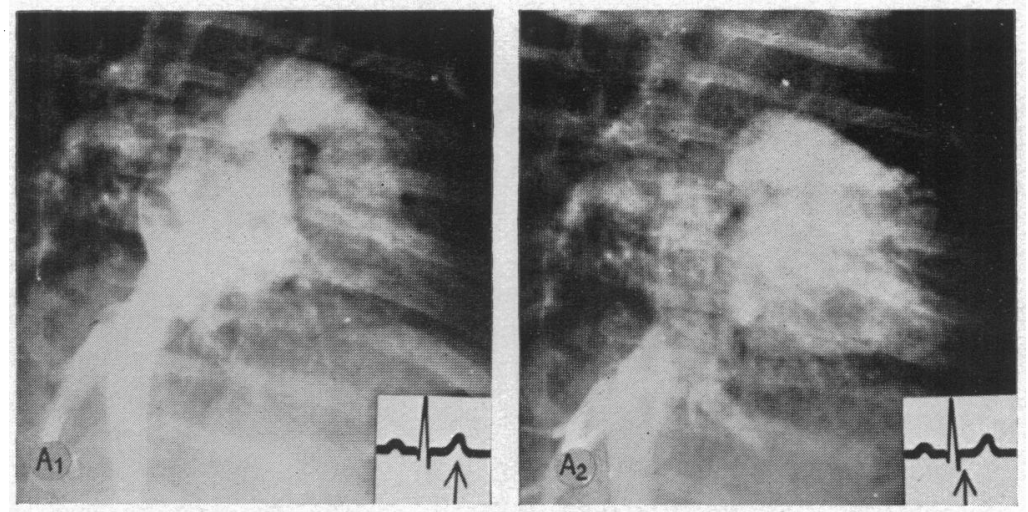

Fig. 5.-Atrial septal defect. Left-to-right shunt. Systolic dilution of contrast in the right atrium. Right (A1-A2) anterior oblique projection.

A1. The dilated right atrium is well filled with contrast in atrial diastole.

A2. In atrial systole the contrast is washed out and the outline of the atrium is lost. Diluted contrast from the atrium has refluxed into the hepatic radicles.

unopacified residual blood and blood from the other vena cava and sinus venosus. On the other hand, a small left-to-right shunt will not be visualized when the right atrium is heavily opacified, because a small jet of radiolucent blood will be invisible in a chamber filled with contrast.

When the contrast material passes through the pulmonary circuit and arrives in the left atrium, 
the right heart is less heavily opacified but is not completely empty of contrast. Here, too, a small stream of blood from left to right will not be observed, even though it is now opacified, because there is not enough difference in opacification.

A patent foramen ovale, of course, will not be visualized when the contrast material reaches the left heart, because this anomaly usually produces a pure right-to-left shunt.

A persistent opacification of the right atrium and ventricle and the pulmonary arteries is presumptive evidence of interatrial communication with a left-to-right shunt, if it can be established that the contrast material in the right heart can have come only from the left. If this is injected slowly or disappears slowly from the afferent vein, the opacification of the right heart is abnormally prolonged, but the source of contrast material is obviously the venous tributaries and not the left heart. Persistent opacification of the right heart can be identified clearly in the left anterior oblique projection.

The consequence of significant left-to-right atrial shunt is dilatation of the right atrium; this results in any chamber subjected to increased filling and increased stroke volume. When this added load interferes with the function of the right atrium, a systolic reflux into the venæ cavæ commonly occurs. This is more often demonstrated in the inferior vena cava, because the orifice of this vein seems to be less securely closed. A retrograde filling of the vena cava caused by forceful injection of the dye is seen in normal patients and has no pathologic significance. However, this usually occurs during diastole when the atrium has become overloaded and before the A-V valves have opened; it may be simulated during systole by a vigorous injection which forces the contrast into the tributary veins when the caval orifice is closed. This retrograde filling may be differentiated from a true systolic reflux by comparing the density of opacification in the atrium, vena cava, and tributary veins. The concentration of the contrast material in the tributary veins is usually approximately equal to that in the vena cava in retrograde diastolic filling, and to that in the atrium in true systolic reflux.

\section{INTERVENTRICULAR SHUNTS (Fig. 6 and 7)}

In the early angiocardiograms, when the right heart is opacified, a significant left-to-right shunt through an interventricular defect causes a dilution of the contrast material in the right ventricle, usually distinctly associated with ventricular systole. The highly opacified right atrium contrasts sharply with the poor concentration in the right ventricle and pulmonary artery. If the defect is high in the interventricular septum, the dilution in the right ventricle is localized to the infundibulum and pulmonary artery.

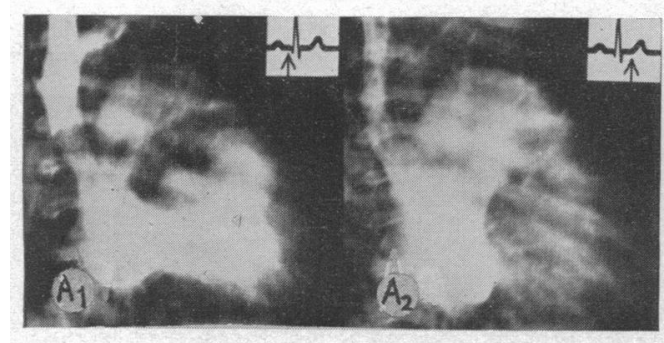

FiG. 6.-Ventricular septal defect. Left-to-right shunt. Systolic dilution in the right ventricle. Right (A1-A2) anterior oblique projection.

A1. Atrial systole fills the right ventricle with concentrated contrast medium.

A2. In ventricular systole the contrast is greatly diluted in the right ventricle by the influx of non-opacified blood from the left ventricle. Note the dilated pulmonary artery.

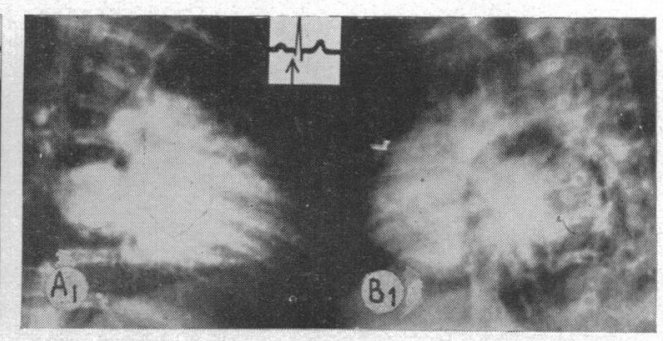

FIG. 7.-Ventricular septal defect. Left-to-right shunt. Prolonged visualization of the right ventricle. Right (A1) and left (B1) anterior oblique projections.

Four seconds after the injection of contrast medium there is a great deal of contrast on both sides of the heart. From A1 it is apparent that there is no opaque substance in the right atrium, and it can be concluded that the contrast in the right ventricle comes from the left ventricle. 
It is sometimes possible to confirm the diagnosis of left-to-right shunt by observing the small amount of opacified blood that remains in the right ventricle at the end of systole: if the dye becomes diluted during systole, this small residual volume becomes invisible.

Prolonged visualization of the right ventricle and pulmonary artery is characteristic of a significant left-to-right shunt. It is of some importance to note the absence of opacification of the right atrium in the right anterior oblique projection, for this will rule out a shunt in the interatrial septum. The angiocardiographic picture is typical: opacification of the right ventricle, pulmonary artery, and left atrium, with poor visualization of the aorta, because the contrast material from the left ventricle is shunted back to the right heart, omitting the systemic circulation. Prolonged opacification of the pulmonary artery is best seen in the right anterior oblique projection, but it is necessary to check the left anterior oblique view to ascertain that the contrast material in the pulmonary artery comes from the right heart and not from the aorta.

In the presence of a left-to-right interventricular shunt, specific adaptations result from the increased stroke volume of the right ventricle; vigorous systolic contraction of the infundibulum and a dilatation of the pulmonary artery most accentuated during ventricular systole, at first functional but later organic. The left atrium is usually strikingly dilated, and occasionally the right atrium is dilated enough to show functional disturbances in the form of refluxes in the inferior vena cava.

\section{Patent Ductus Arteriosus}

Recirculation of blood through the pulmonary artery, indirect evidence of the presence of a patent ductus, is demonstrated by two separate and specific signs, both of which are caused by the shunt of blood from the aorta. The first sign is loss of concentration of contrast material in the pulmonary artery; it occurs early in the angiocardiogram and is the result of the influx of nonopacified blood from the aorta (Fig. 8). This is the most important indirect sign of the presence

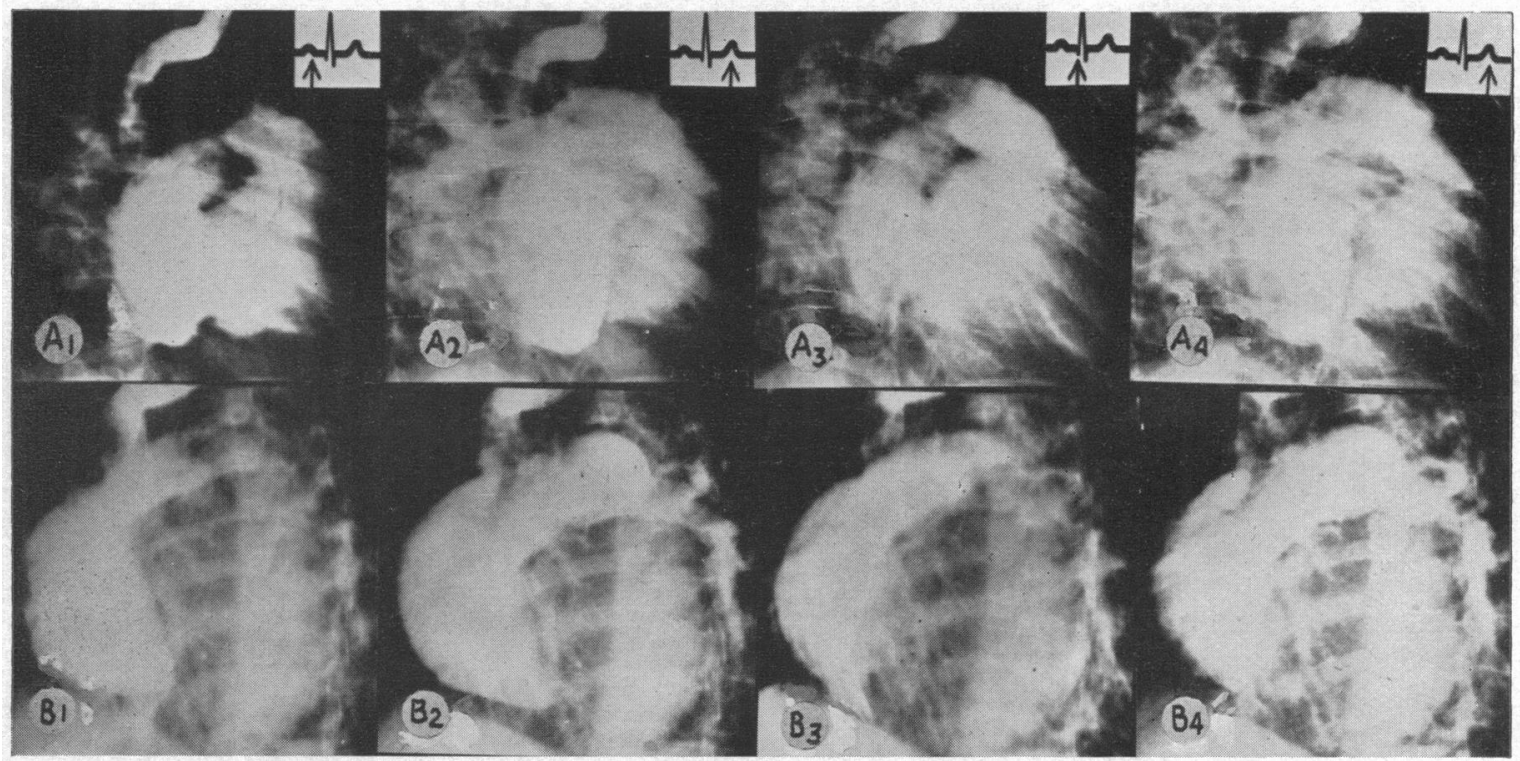

Fig. 8.-Patent ductus. Left-to-right shunt. Diastolic dilution of contrast. Right (A1-A4) and left (B1-B4) anterior oblique projections.

(1) In ventricular diastole the pulmonary artery is only faintly visualized.

(2) In the following ventricular systole the pulmonary artery is heavily opacified. Note the systolic dilatation of the pulmonary artery.

(3) During the succeeding ventricular diastole the contrast material is again washed out of the pulmonary artery.

(4) Ventricular systole once more visualizes the pulmonary artery. 
of a patent ductus and seems to occur with regularity. The dilution reaches a maximum at the end of diastole, because the contrast material is ejected from the right ventricle only during systole; when the pulmonary valve is closed, no additional contrast enters the pulmonary artery. This dilution, most apparent in diastole, rules out a high interventricular septal defect, because in this latter anomaly, the opacification of the pulmonary artery will not change during diastole. The degree of dilution and its progression during the cardiac cycle indicate the size of the shunt. This may also occur in aortic septal defect.

The second indication of recirculation of blood through the pulmonary circuit, persistent visualization of the pulmonary artery, can be expected to occur with regularity in the presence of a patent ductus with a left-to-right shunt, but actually it is rather difficult to demonstrate. By employing a fast injection technique the right and left sides of the heart are normally opacified

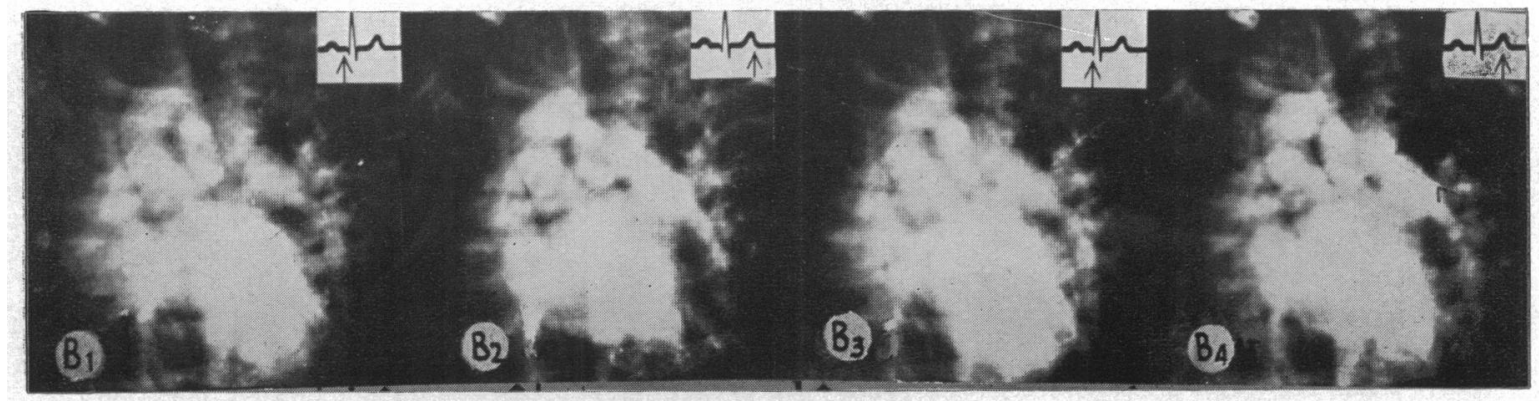

Fig. 9.-Patent ductus. Left-to-right shunt. Re-opacification of the pulmonary artery. Left (B1-B4) anterior oblique projection.

This series of pictures is from the last half of the angiocardiogram, when the right heart is nearly empty of contrast. There is little opacification in the right heart.

(1) In ventricular diastole the pulmonary artery is faintly seen.

(2) In the following ventricular systole the pulmonary artery is densely opacified.

(3) The contrast material in the pulmonary artery is again washed out in ventricular diastole.

(4) The flow of contrast from the aorta again visualizes the pulmonary artery in ventricular systole.

successively, i.e. the right heart and pulmonary artery are relatively empty of contrast material when the concentration is greatest in the aorta (Fig. 9). But when the opacity has reached a maximum in the aorta, there is frequently enough contrast medium remaining in the pulmonary vessels to obscure the introduction of more opacified blood through the patent ductus.

Radiography in two planes simultaneously facilitates the diagnosis of patent ductus. By comparing the two projections, it is possible to state whether there is delayed clearing or repeat opacification of the pulmonary artery, and whether it is revisualized separately or together with the right ventricle (as occurs with interventricular shunt).

\section{SUMMARY}

The intravenous angiocardiographic diagnosis of atrial and ventricular septal defects and patent ductus arteriosus in infants and young children is discussed in detail, both in the case of right-toleft shunts and of left-to-right shunts. Dilution of the opaque medium may often be significant of a shunt, but it can be recognized only if there are frequent angiocardiograms and if the time in the cardiac cycle when they are taken is known. 